\title{
Simplex-like sequential methods for a class of generalized fractional programs
}

\author{
Riccardo Cambini · Laura Carosi . \\ Laura Martein • Ezat Valipour
}

Received: date / Accepted: date

\begin{abstract}
A sequential method for a class of generalized fractional programming problems is proposed. The considered objective function is the ratio of powers of affine functions and the feasible region is a polyhedron, not necessarily bounded. Theoretical properties of the optimization problem are first established and the maximal domains of pseudoconcavity are characterized. When the objective function is pseudoconcave in the feasible region, the proposed algorithm takes advantage of the nice optimization properties of pseudoconcave functions; the particular structure of the objective function allows to provide a simplex-like algorithm even when the objective function is not pseudoconcave. Computational results validate the nice performance of the proposed algorithm.
\end{abstract}

Keywords Generalized fractional programming · Pseudoconcavity · Sequential methods · Global Optimization

Mathematics Subject Classification (2000) 90C05 - 90C31, - 90C32 · 26B25

Riccardo Cambini

Department of Economics and Management, University of Pisa,

Via Ridolfi, 10, 56124 Pisa, Italy E-mail: riccardo.cambini@unipi.it

Laura Carosi

Department of Economics and Management, University of Pisa

E-mail: laura.carosi@unipi.it

Laura Martein

Department of Economics and Management, University of Pisa,

E-mail: lmartein@gmail.com

Ezat Valipour

Department of Mathematics, Shahid Bahonar University of Kerman, Iran

E-mail: ezat.valipour@gmail.com 


\section{Introduction.}

In this paper we will deal with a class of generalized fractional programming problems whose objective function is the ratio of powers of affine functions and whose feasible region is a polyhedron. There is no need to emphasize the importance of fractional programming from both theoretical and applicative point of view (see for all $[8,10-12]$ and references therein).

The stated problem encompasses some particular cases that have been widely studied in fractional programming literature such as the ratio between two affine functions and the ratio of a linear and the power of an affine function (see for instance $[4,5]$ ).

From a computational point of view, the suggested problem is a hard problem since it may have several local maximum points which are not global and the set of optimal solutions may not be convex. For such a reason, we will establish several properties of the objective function related to the existence and the location of the maximum points and to the study of the pseudoconcavity of the objective function. This latter property will be a key tool of our analysis; it is well known that if the objective function is pseudoconcave, then local maximum points are global ones and therefore the problem can be solved more easily with respect to the general case. However, the particular structure of the objective function and the theoretical properties of the problem will allow us to suggest a simplex-like sequential method regardless the objective function is pseudoconcave or not. The proposed algorithm is based on the so called "optimal level solutions" method which has been first conceived for linear fractional problems by Cambini and Martein $[1,2]$ and then applied to solve generalized fractional problems. In his survey, Ellero [9] proposes a unified framework encompassing several contributions of the literature; all of them are based on the "optimal level solutions" method and they successfully solve different classes of generalized fractional problems. More recent analyses, following this approach, have been performed for solving rank-two problems (see for example Cambini Sodini [6]) and rank-three ones (see for example Cambini Sodini [5] and Carosi Martein [7]). At the best of our knowledge, none of the existing contributions deals with the class under analysis in the present paper.

To validate the suggested algorithm, computational tests have been peformed. The algorithm has been implemented with the software MATLAB and various instances have been randomly generated and solved, with a grand total of 520000 problems. Both the pseudoconcave and the non-pseudoconcave case have been considered. Computational results underline that in managing the change of vertex in the iterative steps, the use of simplex table and pivot operations results to be very fast, allowing to solve large problems in a reasonable time.

The paper will be organized as follows: in Section 2 the optimization problem will be stated and its theoretical properties will be studied.

In Section 3 the maximal domain of pseudoconcavity will be investigated, while the idea of the sequential method will be proposed in Section 4. Compu- 
tational results will be discussed in Section 5 and then concluding remarks will be presented in Section 6. A detailed description of the algorithm is proposed in the Appendix.

\section{Statement of the problem and theoretical properties}

Consider the following problem

$$
\begin{aligned}
& P: \sup f(x)=\frac{\left(c^{T} x+c_{0}\right)^{\alpha}}{\left(d^{T} x+d_{0}\right)^{\beta}} \\
& \text { s.t. } x \in X=\left\{x \in \mathbb{R}^{n}: A x=b, x \geq 0\right\} \subset D,
\end{aligned}
$$

where $\alpha>0, \beta>0, D=\left\{x \in \mathbb{R}^{n}: d^{T} x+d_{0}>0\right\}, A$ is a real $m \times n$ matrix, with $\operatorname{rank}[A]=m<n$.

Throughout the paper will make the following assumptions (see Remark 1):

\section{Assumption 1}

i) $c \in \mathbb{R}^{n} \backslash\{0\}$

ii) the parameters $\alpha$ and $\beta$ are specified in order to guarantee the objective function is well defined

iii) $\operatorname{rank}[c, d]=2$.

Remark 1 We are not going to consider $c=0$, because in this case $f$ turns out to be both pseudoconvex and pseudoconcave on $D$ and the sequential methods for this class of functions have been already given (see for all $[3,12]$ and references therein).

As regard the parameters $\alpha$ and $\beta$, note that if $c^{T} x+c_{0} \geq 0$ for every $x \in X$, then the objective function is well defined for every $\alpha>0$. On the other hand, if $c^{T} x+c_{0}$ assumes negative values on $X$, then $\left(c^{T} x+c_{0}\right)^{\alpha}$ is well-defined if and only if $\alpha=\frac{p}{q}, p, q \in \mathbb{N} \backslash\{0\}$, with the greatest common divisor between $p$ and $q$ equal to 1 and $q$ an odd number.

Moreover we assume that $\operatorname{rank}[c, d]=2$, because when the vectors $c$ and $d$ are linearly dependent, it is easy to verify that the behavior of the function reduces to the behavior of a one variable function.

Remark 2 The particular case $\alpha=1$ has been already dealt by Cambini [4], thought no computational tests are present there. The general framework provided by Cambini Sodini in [6] encompasses Problem $\mathrm{P}$ when $c^{T} x+c_{0}$ is positive in the feasible region. Therefore, there is only a partial intersection between this paper and [6].

Remark 3 A very common approach in fractional programming relies on suitable transformations of the objective function which gets the problem easier to be solved; following this approach, one could suggest to consider an objective function where only the numerator or the denominator is the power 
of an affine function, that is, we might consider $\bar{f}(x)=\frac{\left(c^{T} x+c_{0}\right)^{\frac{\alpha}{\beta}}}{d^{T} x+d_{0}}$ or $g(x)=\frac{c^{T} x+c_{0}}{\left(d^{T} x+d_{0}\right)^{\frac{\beta}{\alpha}}}$. Unfortunately this approach is not in general applicable to Problem $\mathrm{P}$ since $c^{T} x+c_{0}$ may change in sign on the feasible region and hence the transformations do not lead to equivalent problems.

Problem P shares some nice theoretical properties with other classes of generalized fractional programming problems (see for example [4,6,7]). Regardless the parameter specifications of function $f$, if an optimal solution exists, it belongs to a feasible edge; furthermore, if the supremum is not attained as a maximum, then there exists an extreme direction along which the function converges to the supremum. The proof of these results uses the following trivial lemma.

Lemma 1 Let $H$ be a subset of $X$.

If $\alpha=\frac{p}{q}$, where $p, q$ are odd numbers or if $c^{T} x+c_{0} \geq 0, \forall x \in H$, then problem $\sup _{x \in H}\left(c^{T} x+c_{0}\right)^{\alpha}$ is equivalent to $L_{1}=\sup _{x \in H}\left(c^{T} x+c_{0}\right)$; in any other case, we have $\sup _{x \in H}\left(c^{T} x+c_{0}\right)^{\alpha}=\sup \left\{L_{1}, L_{2}\right\}$, where $L_{2}=\sup _{x \in H}\left(-c^{T} x-c_{0}\right)$.

We will denote by $L$ the supremum of the Problem $P$.

For the sake of completeness we state and prove the following theorem, though the strategy of its proof is very similar to the ones given for the analogous results in $[4,6,7]$.

Theorem 1 Consider Problem $P$.

i) $L$ is attained as a maximum if and only if there exists a feasible point $x_{0}$ belonging to an edge of $X$ such that $f\left(x_{0}\right)=L$.

ii) If $L$ is not attained as a maximum, then there exists an extreme direction $u$ and a feasible point $x_{0}$ such that $L=\lim _{t \rightarrow+\infty} f\left(x_{0}+t u\right)$.

Proof i) If the supremum $L$ is attained as a maximum, then there exists a feasible point $\bar{x}$ such that $L=f(\bar{x})$. Consider the problem

$$
\bar{P}: \max f(x), x \in \bar{X}=X \cap\left\{x \in \mathbb{R}^{n}: d^{T} x+d_{0}=d^{T} \bar{x}+d_{0}\right\} .
$$

Obviously $\bar{x}$ is an optimal solution of $\bar{P}$ and from Lemma $1, \bar{P}$ is equivalent to a linear problem. Therefore the maximum of $\bar{P}$ is reached at a vertex $x_{0}$ of $\bar{X}$ which necessarily belongs to an edge of $X$. The viceversa is obvious.

ii) Let $\left\{x_{n}\right\} \subset X$ be a sequence which converges to the supremum $L$ and, for any fixed $x_{n}$, consider the following sequence of problems

$$
P_{n}: \sup _{x \in X_{n}} f(x)=\frac{\left(c^{T} x+c_{0}\right)^{\alpha}}{\left(d^{T} x+d_{0}\right)^{\beta}}
$$

where $X_{n}=X \cap\left\{x \in \mathbb{R}^{n}: d^{T} x+d_{0}=d^{T} x_{n}+d_{0}\right\}$. The following two exhaustive cases occur: 
a) there exists $n$ such that the supremum of $P_{n}$ is $+\infty$;

b) for every $n$ the supremum of $P_{n}$ is attained as a maximum.

a) Taking into account Lemma 1, problem $P_{n}$ is equivalent to a suitable linear problem whose feasible region is $X_{n}$, so that there exists a feasible point $x_{0}$ and an extreme direction $u$ along which the objective function goes to $+\infty$. Since it is $d^{T}\left(x_{0}+t u\right)+d_{0}=d^{T} x_{n}+d_{0}$ for every $t \geq 0$, we have $d^{T} u=0$ so that even the supremum of problem $P$ is $+\infty$ and $\lim _{t \rightarrow+\infty} f\left(x_{0}+t u\right)=+\infty$.

b) From Lemma 1, problem $P_{n}$ is equivalent to a suitable linear problem and therefore the maximum is attained at a vertex $y_{n}$ of $X_{n}$ which belongs to an edge of $X$. Taking into account that $f\left(y_{n}\right) \geq f\left(x_{n}\right)$ we have $\lim _{n \rightarrow+\infty} f\left(y_{n}\right)=L$.

Since $X$ has a finite number of edges (in particular half-lines), there exists a subsequence $\left\{\hat{y}_{n}\right\}$ of $\left\{y_{n}\right\}$ contained in an edge of $X$.

Since $L$ is not attained as a maximum, the sequence $\left\{\hat{y}_{n}\right\}$ is necessary divergent in norm and $f\left(\hat{y}_{n}\right) \neq L \forall n$. It follows that $\left\{\hat{y}_{n}\right\}$ is necessarily contained in a half-line $x=x_{0}+t u, t \geq 0$, where $x_{0}$ is a vertex of $X$ and $u$ is an extreme direction. Let $t_{n}$ be such that $\hat{y}_{n}=x_{0}+t_{n} u$. We have $\lim _{n \rightarrow+\infty} f\left(\hat{y}_{n}\right)=\lim _{t_{n} \rightarrow+\infty} f\left(x_{0}+t_{n} u\right)=L$.

Whenever $L$ is not attained as a maximum and $\alpha \neq \beta$, the supremum is obviously either 0 or $+\infty$. This fact is strictly related to the particular structure of the objective function $f$. The following theorem completely characterizes the value of $L$ for Problem P.

Theorem 2 Consider Problem $P$.

Assume that the supremum $L$ is not attained as a maximum and $\alpha \neq \beta$.

i) $L=0$ if and only if $f(x)<0$ for every $x \in X$ and there exists an extreme direction $u$ such that either $d^{T} u>0$ and $\alpha<\beta$, or $c^{T} u=0$ and $\alpha>\beta$.

ii) $L=+\infty$ if and only if $f$ assumes some positive values on $X$ and there exists an extreme direction $u$ such that either $d^{T} u=0$ and $\alpha<\beta$, or $c^{T} u \neq 0$ and $\alpha>\beta$.

Proof From i) of Theorem 1, there exists an extreme direction $u$ such that $L=\lim _{t \rightarrow+\infty} \frac{\left(c^{T} x_{0}+t c^{T} u+c_{0}\right)^{\alpha}}{\left(d^{T} x_{0}+t d^{T} u+d_{0}\right)^{\beta}}$. Since $L$ is not attained as a maximum, $c^{T} u$ and $d^{T} u$ cannot be contemporarily equal to zero. i) and ii) follow by computing the limit.

Remark 4 Note that, when $\alpha=\beta$, we have

$$
L=\lim _{t \rightarrow+\infty} \frac{\left(c^{T} x_{0}+t c^{T} u+c_{0}\right)^{\alpha}}{\left(d^{T} x_{0}+t d^{T} u+d_{0}\right)^{\beta}}=\lim _{t \rightarrow+\infty}\left(\frac{c^{T} x_{0}+t c^{T} u+c_{0}}{d^{T} x_{0}+t d^{T} u+d_{0}}\right)^{\alpha}
$$

so that $L$ can assume any value, finite or not. 
3 On the maximal domains of pseudoconcavity

As we have just outlined, a key tool of our analysis is the pseudoconcavity of the objective function. It is well known that if the objective function is pseudoconcave, then a critical point or a local maximum point is also a global one and therefore Problem $\mathrm{P}$ can be solved more easily with respect to the general case.

In what follows $\nabla f(x)$ and $H(x)$ will denote the gradient and the Hessian matrix of $f$ evaluated at $x$, respectively.

For the sake of completeness, we recall the definition of a pseudoconcave function.

Definition 1 Let $f$ be a real-valued differentiable function defined on a convex set $C \subseteq \mathbb{R}^{n}$. $f$ is said to be pseudoconcave on $C$ if and only if

$$
\forall x_{1}, x_{2} \in C, \quad f\left(x_{1}\right)<f\left(x_{2}\right) \Rightarrow\left(x_{2}-x_{1}\right)^{T} \nabla f\left(x_{1}\right)>0 .
$$

In order to characterize the pseudoconcavity of $f$, we will use the following second order characterization (see for all [3]).

Theorem 3 Let $f$ be a twice differentiable function defined on an open convex set $O \subseteq \mathbb{R}^{n}$. Then, $f$ is pseudoconcave on $O$ if and only if the following two conditions hold:

i) $x \in O, v \in \mathbb{R}^{n}, v^{T} \nabla f(x)=0 \Rightarrow v^{T} H(x) v \leq 0$;

ii) If $x_{0} \in O$ is a critical point, then $x_{0}$ is a local maximum point for $f$ on $O$.

According to Theorem 3, we have to analyze the behavior of the Hessian matrix on the directions which are orthogonal to the gradient and we have to establish whether the critical points are maximum points. With respect to the introduced fractional function $f(x)=\frac{\left(c^{T} x+c_{0}\right)^{\alpha}}{\left(d^{T} x+d_{0}\right)^{\beta}}$, we get:

$$
\begin{gathered}
\nabla f(x)=\frac{\left(c^{T} x+c_{0}\right)^{\alpha-1}}{\left(d^{T} x+d_{0}\right)^{\beta+1}}\left(\left(d^{T} x+d_{0}\right) \alpha c-\left(c^{T} x+c_{0}\right) \beta d\right) \\
H(x)=\frac{\left(c^{T} x+c_{0}\right)^{\alpha-2}}{\left(d^{T} x+d_{0}\right)^{\beta+2}}\left(\left(d^{T} x+d_{0}\right)^{2}\left(\alpha^{2}-\alpha\right) c c^{T}-\right. \\
\left.\alpha \beta\left(c^{T} x+c_{0}\right)\left(d^{T} x+d_{0}\right)\left(c d^{T}+d c^{T}\right)+\left(c^{T} x+c_{0}\right)^{2}\left(\beta^{2}+\beta\right) d d^{T}\right)
\end{gathered}
$$

Let us preliminary observe that the only points where $f$ might not be differentiable are the zeros of the function. Moreover, whenever $f$ is differentiable, the linear independence of $c$ and $d$ implies that the critical points of the function coincide with its zeros. Whenever $f$ assumes some positive values, the zeros of the functions are not maximum points and therefore $f$ is not pseudoconcave. On the other hand, in every subset of $D$ where $f$ is not positive, then the zero points are the maximum points. In this light, we will study the maximal domains of pseudoconcavity of $f$ on the sets $S^{+}=\{x \in D: f(x)>0\}$ and $S^{-}=\{x \in D: f(x)<0\}$. Consider the sets $C^{+}=S^{+} \cap\left\{x \in \mathbb{R}^{n}: c^{T} x+c_{0}>0\right\}$ and $C^{-}=S^{+} \cap\left\{x \in \mathbb{R}^{n}: c^{T} x+c_{0}<0\right\}$; the following theorem holds. 
Theorem 4 Consider function $f$ together with Assumptions 1.

The following statements hold:

i) $f$ is pseudoconcave on $S^{-}$if and only if $\alpha \geq \beta$.

ii) $f$ is pseudoconcave on $C^{+}$and on $C^{-}$if and only if $\alpha \leq \beta$.

Proof Let us first observe that the linear independence of vectors $c$ and $d$ implies that $f$ has no critical points on $S^{+}$and $S^{-}$. Referring to condition i) in Theorem 3, let $v \neq 0$ be a direction such that $\nabla f(x)^{T} v=0$; we have

$$
\begin{gathered}
c^{T} v=\frac{\beta}{\alpha} \frac{\left(c^{T} x+c_{0}\right)}{\left(d^{T} x+d_{0}\right)} d^{T} v, \\
v^{T} H(x) v=\frac{\beta}{\alpha} \frac{\left(c^{T} x+c_{0}\right)^{\alpha}}{\left(d^{T} x+d_{0}\right)^{\beta+2}}(\alpha-\beta)\left(d^{T} v\right)^{2} .
\end{gathered}
$$

Consequently $v^{T} H(x) v \leq 0$ for every $x \in S^{-}$if and only if $\alpha-\beta \geq 0$, and i) holds.

Consider now the set $S^{+} ; v^{T} H(x) v \leq 0$ for every $x \in S^{+}$if and only if $\alpha-\beta \leq 0$. If there exists $x \in D$ such that $c^{T} x+c_{0}=0$, then $S^{+}$may be not convex, and hence we consider the convex open sets $C^{+}$and $C^{-}$. If either $C^{+}=\emptyset$ or $C^{-}=\emptyset$, then the thesis immediately follows. In the case $C^{+}$ and $C^{-}$are both non-empty, i.e. $\left\{x \in \mathbb{R}^{n}: c^{T} x+c_{0}=0\right\} \cup S^{+}=D, f$ is pseudoconcave on the two convex sets $C^{+}$and $C^{-}$if and only if $\alpha \leq \beta$.

The particular structure of function $f$ allows us to easily characterize the maximal domains of the pseudoconvexity too.

Theorem 5 Consider function $f$ together with Assumptions 1.

The following statements hold:

i) $f$ is pseudoconvex on $C^{+}$and on $C^{-}$if and only if $\alpha \geq \beta$.

ii) $f$ is pseudoconvex on $S^{-}$if and only if $\alpha \leq \beta$.

Proof It is sufficient to recall that $f$ is pseudoconvex if and only if $-f$ is pseudoconcave.

Remark 5 Observe that when $\alpha=\beta, f$ is pseudolinear on the sets $S^{-}, C^{+}$ and $C^{-}$.

Theorem 5 allows us to specify i) of Theorem 1 for some subclasses of the problem.

Theorem 6 Assume one of the following conditions holds:

i) $\alpha \leq \beta$ and $X \subset S^{-}$;

ii) $\alpha \geq \beta$ and $X \subset C^{+}$;

iii) $\alpha \geq \beta$ and $X \subset C^{-}$

If the supremum of problem $P$ is attained as a maximum, then there exists a vertex of $X$ which is a maximum point.

Proof From Theorem 5, $f$ is pseudoconvex on $X$. The result follows from the properties of pseudoconvex functions (see for all [3]). 


\section{Sequential method}

The proposed sequential method distinguishes the case where $f$ is pseudoconcave on the feasible region $X$ from the case where $f$ is not pseudoconcave on $X$. In the first case, local optimal solutions are global ones and this property allows us to solve problem $\mathrm{P}$ very fast, while in the second case we have to keep track of all the local optimal solutions the algorithm finds. In both cases, the procedure benefits from the particular structure of the objective function, it splits the feasible region $X$ and, consistently, it separately looks for the optimal points in the following subsets of $X$ (of course, some of them might be empty):

1. $X_{>}=\left\{x \in X: c^{T} x+c_{0} \geq 0\right\}$, that is the set where the numerator is non-negative;

2. $X_{<}^{+}=\left\{x \in X: c^{T} x+c_{0} \leq 0, f(x) \geq 0\right\}$, that is the set of feasible points where the numerator is non-positive and function $f$ is non-negative;

3. $X_{<}^{-}=\left\{x \in X: c^{T} x+c_{0} \leq 0, f(x) \leq 0\right\}$, that is the set where function $f$ is non-positive.

Looking for the optimal solution of $P$ restricted to $X_{>}$is equivalent to look for the optimal solution on $X \cap C^{+}$; similarly, if a maximum point of $f$ on $X_{<}^{+}$exists, it belongs to $X \cap C^{-}$and it is also the maximum of the function $\frac{\left(-c^{T} x-c_{0}\right)^{\alpha}}{\left(d^{T} x+d_{0}\right)^{\beta}}$ on $X \cap C^{-}$. Moreover, whenever there exists a zero of $f$ on $X_{<}^{-}$ this is obviously the maximum point and no further analysis is needed; if $f$ is always negative on $X_{<}^{-}$, then $X_{<}^{-}=X \cap S^{-}$. Therefore, once we have preliminarly checked that the maximum point is not a zero of $f$, we can restrict our analysis on the sets $C^{+}, C^{-}$and $S^{-}$. This restriction is extremely helpful since, on those sets $f$ is either pseudoconcave or pseudoconvex (see Section 3 ). Of course either $S^{-} \neq \emptyset$ or $C^{+} \cup C^{-} \neq \emptyset$ and in case both $C^{+} C^{-}$are nonempty, the maximum point of $f$ will be computed by comparing the maximum value of $f$ on $C^{+}, C^{-}$. The procedures which find the maximum points of $f$ on $C^{+}$, $C^{-}$and $S^{-}$are based on the "optimal level solutions" method introduced by $[1]$.

Roughly speaking, the basic idea of this method consists in "slicing" the feasible region according to the possible values the denominator may assume. For each value of the denominator, the maximum of a suitable function (in most cases the numerator) is computed; this is done by solving a parametric problem where the parameter varies according to the value of the denominator on the feasible region. For each value of the parameter, the solutions of the parametric problem are called "optimal level solutions" and the maximum value of the original function is found among them. Quoting [2], the algorithm "generates a sequence of finite optimal level solutions $x^{1}, x^{2} \ldots, x^{k}$, which corresponds to increasing levels of the original objective function"; then either the maximum point does not exist or it coincides with the last point of the sequence". This approach has been first conceived for linear fractional problems and then applied to suggest several sequential methods for generalized fractional programming (see for example Ellero [9] and Cambini Martein [2] 
and references therein, Cambini Sodini [5,6] and Carosi Martein [7]).

Referring to problem $\mathrm{P}$, it is worth noticing that the denominator function $d^{T} x+d_{0}$ is lower bounded on $X$, so that the linear problem

$$
P_{d}: \min _{x \in X}\left(d^{T} x+d_{0}\right)
$$

has optimal solutions. Let $\theta_{\min }$ be the minimum value of $P_{d}$.

Consider now the linear program

$$
P_{\bar{c}}: \max \left\{\bar{c}^{T} x+\bar{c}_{0}, x \in K \cap\left\{x: d^{T} x+d_{0}=\theta_{\min }\right\}\right.
$$

where $K$ can be $C^{+}$or $C^{-}$or $S^{-}$and

$$
\bar{c}^{T} x+\bar{c}_{0}=\left\{\begin{array}{l}
c^{T} x+c_{0} \quad \text { if } K=C^{+} \text {or } K=S^{-} \\
-c^{T} x-c_{0} \text { if } K=C^{-}
\end{array}\right.
$$

Assume that the supremum of $P_{\bar{c}}$ is finite and let $x_{0}$ be a vertex of $X$ which is an optimal solution of $P_{\bar{c}}$. Starting from $x_{0}$, we suggest an algorithm for determine a local maximum point (if one exists) for problem $P$.

Consider the linear parametric problem

$$
P(\theta): \psi(\theta)=\max \left(\bar{c}^{T} x+\bar{c}_{0}, x \in K(\theta)=K \cap\left\{x: d^{T} x+d_{0}=\theta_{\min }+\theta\right\}\right.
$$

where $\theta \in \Theta=\{\theta: K(\theta) \neq \emptyset\}=\left[0, \theta_{\max }-\theta_{\min }\right]$, and $\theta_{\max }$ may be $+\infty$.

We have

$$
\max _{x \in K} f(x)=\max _{\theta \in \Theta} \max _{x \in K(\theta)} f(x) .
$$

Setting $h(\theta)=\max _{x \in K(\theta)} f(x)$, it results

$$
\max _{x \in K} f(x)=\max _{\theta \in \Theta} h(\theta), h(\theta)=\frac{\psi(\theta)^{\alpha}}{\left(\theta_{\min }+\theta\right)^{\beta}} .
$$

If $h$ increases (decreases), then the function $f$ increases (decreases) so that a local maximum of $h$ corresponds to a local maximum of $f$. The sequential method determines a local maximum point for $h$ (if one exists).

The idea is the following: initialize the procedure with $\theta_{0}=0$ so that the vertex $x_{0}$ is an optimal solution of $P\left(\theta_{0}\right)$, then denote by $B_{0}$ the set of indices associated with the basic variables and set $x_{0}=\left(x_{B_{0}}, 0\right)$. It's worth remarking that, with respect to problem $P, P(\theta)$ has the additional constraint $d^{T} x+d_{0}=$ $\theta_{\min }+\theta$. This leads to the introduction of an additional slack variable $x_{n+1}$. According to the idea of the "optimal level solutions" method, for any value of $\theta$, every optimal solution of $P(\theta)$ is binding to the parametric constraint, so that there exists a basic optimal solution $\left(x_{B_{0}}, 0\right)$ such that the variable $x_{n+1}$ is non-basic. With a little abuse of notation, we will refer to $\left(x_{B_{k}}, 0\right)$ as a basic solution of $P$. Applying sensitivity analysis we find $\left(x_{B_{0}}(\theta), 0\right)=\left(x_{B_{0}}+\right.$ $\left.\theta u_{B_{0}}, 0\right)$ which is optimal for $P(\theta)$ for every $\theta$ belonging to the stability interval $\left[\theta_{0}, \theta_{1}\right]=\left\{\theta: x_{B_{0}}(\theta) \geq 0\right\}$. If $h^{\prime}\left(\theta_{0}\right) \leq 0$, then $\left(x_{B_{0}}, 0\right)$ is a local maximum for $h$ and it corresponds to a local maximum point for $P$. If $h^{\prime}\left(\theta_{0}\right)>0$ and there 
exists $\widetilde{\theta} \in\left[\theta_{0}, \theta_{1}\right]$ such that $h^{\prime}(\widetilde{\theta})=0$, then $\left(x_{B_{0}}(\widetilde{\theta}), 0\right)$ is a local maximum point for $h$ and it corresponds to a local maximum for $P$. In this latter case the local maximum point of $P$ belongs to an edge of $X$. In any other case, for $\theta>\theta_{1}$ the feasibility is lost and it is restored by applying an iteration of the dual simplex algorithm. We find a new stability interval and we repeat the analysis. Proceeding in this way we develop a finite sequence of basis $B_{k}, k=0,1, \ldots$ and a finite numbers of stability intervals $\left[\theta_{k}, \theta_{k+1}\right], k=0,1, \ldots$.

With the usual notations, corresponding to the basis $B_{k}$, we have: $x(\theta)=\left(x_{B_{k}}(\theta), 0\right)=\left(x_{B_{k}}+\theta u_{B_{k}}, 0\right), \quad \psi(\theta)=\bar{c}_{B_{k}}^{T} x_{B_{k}}+\theta \bar{c}_{B_{k}}^{T} u_{B_{k}}+\bar{c}_{0}, \theta \in$ $\left[\theta_{k}, \theta_{k+1}\right]$ so that

$$
\begin{gathered}
h(\theta)=\frac{\left(\bar{c}_{B_{k}}^{T} x_{B_{k}}+\theta \bar{c}_{B_{k}}^{T} u_{B_{k}}+\bar{c}_{0}\right)^{\alpha}}{\left(\theta_{\min }+\theta\right)^{\beta}}, \theta \in\left[\theta_{k}, \theta_{k+1}\right] \\
h^{\prime}(\theta)=\frac{\left(\bar{c}_{B_{k}}^{T} x_{B_{k}}+\theta \bar{c}_{B_{k}}^{T} u_{B_{k}}+\bar{c}_{0}\right)^{\alpha-1}}{\left(\theta_{\min }+\theta\right)^{\beta+1}}\left(\theta(\alpha-\beta) \bar{c}_{B_{k}}^{T} u_{B_{k}}+\xi_{B_{k}}\right), \theta \in\left[\theta_{k}, \theta_{k+1}\right]
\end{gathered}
$$

where $\xi_{B_{k}}=\alpha \bar{c}_{B_{k}}^{T} u_{B_{k}} \theta_{\min }-\beta\left(\bar{c}_{B_{k}}^{T} x_{B_{k}}+\bar{c}_{0}\right)$.

When $f$ is pseudoconcave (see subprocedure Visit1 in the Appendix), a local maximum point $\widehat{\theta}_{k}$ of $h$ corresponds to a global maximum for $P$. In the pseudoconvex case (see subprocedure Visit2 in the Appendix), we look for another value $\widetilde{\theta}_{k}$ of $\theta$ such that $h\left(\widetilde{\theta}_{k}\right)=h\left(\widehat{\theta}_{k}\right)$. The uniqueness of $\widetilde{\theta}_{k}>0$ is guaranteed by the pseudoconvexity of $h$, together with $\lim _{\theta \rightarrow+\infty} h(\theta)=0$ (if $\alpha<\beta$ ) or $\lim _{\theta \rightarrow+\infty} h(\theta)=+\infty$ (if $\alpha>\beta$ ). We make a jump setting $d^{T} x+d_{0}=\theta_{\min }+\widetilde{\theta}_{k}$ so that the procedure goes on by considering the following parametric problems:

$\widetilde{P}(\theta): \psi(\theta)=\max \left(\bar{c}^{T} x+\bar{c}_{0}\right), x \in X(\theta)=X \cap\left\{x: d^{T} x+d_{0}=\theta_{\min }+\widetilde{\theta}_{k}+\theta\right\}$

where $\theta \in\left[0, \theta_{\max }-\widetilde{\theta}_{k}-\theta_{\min }\right]$.

Observe that if $\widetilde{P}(\theta)$ does not have solutions, then $x\left(\widehat{\theta}_{k}\right)$ is a global maximum point for $P$.

The following example aims at clarifying the idea of the procedure, pointing out that the analyzed problem may admit several local maximum points which are not global ones and that the algorithm is able to find the global maximum point. In the Appendix, the whole algorithm will be described in detail.

Example 1 Consider the function $f(x)=\frac{\left(x_{1}+x_{2}+1\right)^{3}}{\left(3 x_{1}+2 x_{2}+1\right)^{2}}$ and the feasible region $X=\left\{x \in \mathbb{R}^{2}: x_{1}+6 x_{2} \leq 30,3 x_{1}+8 x_{2} \leq 45, x_{1} \geq 0, x_{2} \geq 0\right\}$.

Let's preliminary observe that the numerator is positive on $\mathrm{X}$, so that $X=C^{+}$; we get $\theta_{\min }=1$ and $\theta_{\max }=45$. Consider the parametric problem

$$
P_{\theta}: \max \left\{x_{1}+x_{2}+1, x \in C^{+} \cap\left\{x: 3 x_{1}+2 x_{2} \leq \theta\right\}\right.
$$

The point $(0,0)$ is the optimal level solution for $P_{0}$ and the associate basic solution is $x(\theta)=\left(0, \frac{1}{2} \theta, 30-3 \theta, 45-\theta, 0\right)^{T}$ whose stability interval is $\mathcal{F}=[0,10]$. 
We get $h(\theta)=\frac{\left(-1-\frac{1}{2} \theta\right)^{3}}{(\theta+1)^{2}} \cdot \theta=0$ is a local maximum point for $h$ and hence $(0,0)$ is local maximum for $f$ with $f(0,0)=1$. Looking for a point such that $h(\theta)=h(0)$ we find $\tilde{\theta}=1+\sqrt{5} \in \mathcal{F}$; therefore, we go to the adjacent vertex $(0,5)$ whose associated basis is $x(\theta)=\left(\frac{3}{8} \theta-\frac{15}{4}, \frac{45}{8}-\frac{1}{16} \theta, 0, \frac{45}{4} \theta-\frac{5}{8} \theta, 0\right)$. The incumbent optimal value is now $f(0,5)=1.785$. The new stability interval is $\mathcal{F}=[10,18]$ and we get $h(\theta)=\frac{\left(\frac{23}{8}+\frac{5}{16} \theta\right)^{3}}{(\theta+1)^{2}}$. As $h^{\prime}(\theta)>0$ for $\theta>\frac{77}{5} \in \mathcal{F}, \theta=10$ is a local maximum point for $h ; h$ assumes the value $h(10)$ even in $\tilde{\theta} \simeq 23.9073 \notin \mathcal{F}$ and $\tilde{\theta}<\theta_{\max }$. By performing an iteration of the dual algorithm we restore feasibility and we obtain the basic solution $x(\theta)=\left(5.6255+\frac{4}{9} \theta, 3.5154-\frac{1}{6} \theta, \frac{5}{9} \theta+3.2819 \theta, 0,0\right)$. The updated value of $\theta_{\max }$ is $45-23.9073=21.0927$ and the new stability interval is $\mathcal{F}=[0,21.0927]$. The updated function $h$ is $h(\theta)=\frac{\left(10.141+\frac{5}{18} \theta\right)^{3}}{(\theta+24.9073)^{2}}$ which is increasing for $\theta \geq 0$. Therefore the vertex corresponding to $\theta=21.0926=\theta_{\max }$ is a local maximum point whose corresponding value is 1.9357 . Since we have reached $\theta_{\max }$ and $1.9357>1.785$, the vertex $(15,0)$ is the optimal solution for Problem $\mathrm{P}$.

\section{Computational results}

The previously described procedures have been fully implemented with the software MATLAB 8.5 R2015a on a iMac OSX Yosemite computer having 16 Gb RAM and an i7 quad core processor at $3.5 \mathrm{GHz}$. Within the procedures, the linear problems have been solved by using the Gurobi 6.0 or the Cplex 12.6 numerical engines.

Various instances have been randomly generated and solved, with a grand total of 520000 problems solved.

Six different pairs of $\alpha$ and $\beta$ have been used to consider pseudoconcavity and/or pseudoconvexity properties.

Matrix $A \in \mathbb{R}^{m \times n}$ and vectors $c, d \in \mathbb{R}^{n}, b \in \mathbb{R}^{m}$ have been randomly generated with components in the interval $[-10,10]$ by using the "randi()" MATLAB function (integer numbers generated with uniform distribution). The value $d_{0} \in \mathbb{R}$ has been chosen in order to have function $d^{T} x+d_{0}$ positive over the feasible region. Three different values of $c_{0} \in \mathbb{R}$ have been chosen in order to have function $c^{T} x+c_{0}$ either positive or negative or changing sign over the feasible region.

The results of the computational test are collected in the following three tables. The provided values tell how long the algorithm worked to solve the various instances for the various considered cases. Specifically speaking, Table 1 provides the needed average number of iterations, Table 2 provides the average spent CPU times (given by the "cputime" MATLAB command), Table 3 
provides the average spent real times (given by the "tic" and "toc" MATLAB commands). In this light, notice that:

- " $X_{>}$" denotes the case of regions $X$ such that $c^{T} x+c_{0} \geq 0 \forall x \in X$;

- " $X<_{<}$" denotes the case of regions $X$ such that $c^{T} x+c_{0} \leq 0 \forall x \in X$;

- " $X<>$ " denotes the case of regions $X$ where $\exists x_{1}, x_{2} \in X$ such that $c^{T} x_{1}+$ $c_{0}<0$ and $c^{T} x_{2}+c_{0}>0$

- " $m \times n$ " represents the dimension of matrix $A$ in the considered problems;

- "num" represents the number of randomly generated problems solved for the corresponding dimension $m \times n$;

- the pairs of $\alpha$ and $\beta$ provide the parameters chosen for the objective function.

Table 1 Computational Results - Number of Iterations

\begin{tabular}{|c|c|c|c|c|c|c|c|c|}
\hline$X$ & $m \times n$ & num & $\begin{array}{l}\alpha=2 \\
\beta=3\end{array}$ & $\begin{array}{l}\alpha=3 \\
\beta=5\end{array}$ & $\begin{array}{l}\alpha=1 / 3 \\
\beta=3 / 4\end{array}$ & $\begin{array}{l}\alpha=3 \\
\beta=2\end{array}$ & $\begin{array}{l}\alpha=5 \\
\beta=3\end{array}$ & $\begin{array}{l}\alpha=1 / 2 \\
\beta=1 / 4\end{array}$ \\
\hline \multirow{9}{*}{$X_{>}$} & $60 \times 80$ & 10000 & 14.975 & 13.992 & 11.449 & 24.09 & 25.117 & 26.693 \\
\hline & $90 \times 120$ & 10000 & 28.41 & 26.67 & 22.055 & 42.529 & 43.946 & 46.222 \\
\hline & $120 \times 160$ & 6000 & 45.02 & 42.513 & 35.508 & 64.054 & 65.963 & 68.801 \\
\hline & $150 \times 200$ & 3000 & 63.738 & 60.41 & 51.052 & 87.237 & 89.418 & 92.587 \\
\hline & $180 \times 240$ & 1500 & 85.995 & 81.829 & 69.635 & 114.65 & 117.76 & 121.8 \\
\hline & $210 \times 280$ & 800 & 110.78 & 105.8 & 90.756 & 144.72 & 148.09 & 152.98 \\
\hline & $240 \times 320$ & 500 & 138.3 & 132.38 & 114.69 & 176.44 & 181.3 & 185.17 \\
\hline & $270 \times 360$ & 400 & 167.78 & 160.55 & 139.71 & 212.22 & 216.62 & 222.72 \\
\hline & $300 \times 400$ & 300 & 187.81 & 180.02 & 157.7 & 235.72 & 239.42 & 247.73 \\
\hline \multirow{9}{*}{$X_{<}$} & $60 \times 80$ & 10000 & 14.96 & 34.227 & 11.448 & 33.774 & 33.752 & $\mathrm{~N} / \mathrm{A}$ \\
\hline & $90 \times 120$ & 10000 & 28.205 & 56.751 & 21.933 & 56.193 & 56.169 & $\mathrm{~N} / \mathrm{A}$ \\
\hline & $120 \times 160$ & 6000 & 44.93 & 82.066 & 35.474 & 81.491 & 81.464 & $\mathrm{~N} / \mathrm{A}$ \\
\hline & $150 \times 200$ & 3000 & 64.85 & 109.45 & 51.81 & 108.74 & 108.72 & $\mathrm{~N} / \mathrm{A}$ \\
\hline & $180 \times 240$ & 1500 & 87.719 & 140.96 & 71.106 & 140.34 & 140.31 & $\mathrm{~N} / \mathrm{A}$ \\
\hline & $210 \times 280$ & 800 & 111.45 & 175.05 & 91.269 & 174.15 & 174.1 & $\mathrm{~N} / \mathrm{A}$ \\
\hline & $240 \times 320$ & 500 & 140.14 & 211.23 & 115.48 & 210.26 & 210.19 & $\mathrm{~N} / \mathrm{A}$ \\
\hline & $270 \times 360$ & 400 & 166.16 & 251.62 & 139.91 & 250.27 & 250.22 & $\mathrm{~N} / \mathrm{A}$ \\
\hline & $300 \times 400$ & 300 & 197.39 & 277.1 & 165.33 & 275.91 & 275.85 & $\mathrm{~N} / \mathrm{A}$ \\
\hline \multirow{9}{*}{$X_{<>}$} & $60 \times 80$ & 10000 & 40.655 & 19.518 & 33.886 & 26.337 & 26.874 & $\mathrm{~N} / \mathrm{A}$ \\
\hline & $90 \times 120$ & 10000 & 73.385 & 35.427 & 62.572 & 45.484 & 46.184 & $\mathrm{~N} / \mathrm{A}$ \\
\hline & $120 \times 160$ & 6000 & 112.99 & 54.776 & 98.049 & 67.763 & 68.682 & $\mathrm{~N} / \mathrm{A}$ \\
\hline & $150 \times 200$ & 3000 & 157.74 & 76.171 & 138.72 & 92.211 & 93.495 & $\mathrm{~N} / \mathrm{A}$ \\
\hline & $180 \times 240$ & 1500 & 209.53 & 101.64 & 186.31 & 120.93 & 122.08 & $\mathrm{~N} / \mathrm{A}$ \\
\hline & $210 \times 280$ & 800 & 264.98 & 129.45 & 237.45 & 151.82 & 153.27 & $\mathrm{~N} / \mathrm{A}$ \\
\hline & $240 \times 320$ & 500 & 327.87 & 159.02 & 295.38 & 184.1 & 185.21 & $\mathrm{~N} / \mathrm{A}$ \\
\hline & $270 \times 360$ & 400 & 390.28 & 192.71 & 354.08 & 221.26 & 222.92 & $\mathrm{~N} / \mathrm{A}$ \\
\hline & $300 \times 400$ & 300 & 442.73 & 213.75 & 403.29 & 243.59 & 245.71 & $\mathrm{~N} / \mathrm{A}$ \\
\hline
\end{tabular}

The obtained results point out the behavior of the solution algorithm with respect to the problems dimension. In particular, it is worth noticing that:

- the algorithm utilizes the generalized concavity/convexity property of the objective function as a stopping criterion thus improving the performance;

- the algorithm manages real parameters $\alpha$ and $\beta$, and the results confirm that the solution method works well with rational parameters $\alpha$ and $\beta$;

- the use of simplex table and pivot operations in managing the change of vertex iterative steps results to be very fast, allowing to solve large problems in a reasonable time. 
Table 2 Computational Results - CPU Times (secs)

\begin{tabular}{|c|c|c||c|c|c|c|c|c|}
\hline \multirow{3}{*}{} & $m \times n$ & $n u m$ & $\beta=3$ & $\beta=5$ & $\beta=3 / 4$ & $\beta=2$ & $\beta=3$ & $\beta=1 / 4$ \\
\hline \hline \multirow{6}{*}{$X_{>}$} & $60 \times 80$ & $10^{4}$ & 0.12946 & 0.12595 & 0.10419 & 0.21701 & 0.22619 & 0.23989 \\
& $90 \times 120$ & $10^{4}$ & 0.59795 & 0.56572 & 0.46822 & 0.89632 & 0.92548 & 0.97628 \\
& $120 \times 160$ & 6000 & 1.881 & 1.7805 & 1.4852 & 2.6598 & 2.7401 & 2.8663 \\
& $150 \times 200$ & 3000 & 4.3986 & 4.1721 & 3.5202 & 5.9859 & 6.1353 & 6.3704 \\
& $180 \times 240$ & 1500 & 9.5089 & 9.048 & 7.699 & 12.608 & 12.951 & 13.422 \\
& $210 \times 280$ & 800 & 19.676 & 18.766 & 16.05 & 25.58 & 26.204 & 27.12 \\
& $240 \times 320$ & 500 & 38.797 & 37.117 & 32.203 & 49.312 & 50.63 & 51.887 \\
& $270 \times 360$ & 400 & 58.221 & 55.555 & 48.417 & 73.319 & 74.812 & 77.085 \\
& $300 \times 400$ & 300 & 98.047 & 93.7 & 82.047 & 122.73 & 124.62 & 129.06 \\
\hline \multirow{5}{*}{$X_{<}$} & $60 \times 80$ & $10^{4}$ & 0.13449 & 0.29982 & 0.10439 & 0.29546 & 0.2956 & N/A \\
& $90 \times 120$ & $10^{4}$ & 0.59791 & 1.2012 & 0.46498 & 1.1903 & 1.1896 & N/A \\
& $120 \times 160$ & 6000 & 1.8816 & 3.4383 & 1.4832 & 3.4173 & 3.4167 & N/A \\
& $150 \times 200$ & 3000 & 4.4548 & 7.5747 & 3.5571 & 7.5319 & 7.5308 & N/A \\
& $180 \times 240$ & 1500 & 9.7143 & 15.622 & 7.8548 & 15.564 & 15.56 & N/A \\
& $210 \times 280$ & 800 & 19.862 & 31.147 & 16.255 & 31.019 & 31.038 & N/A \\
& $240 \times 320$ & 500 & 39.485 & 59.338 & 32.505 & 59.067 & 59.077 & N/A \\
& $270 \times 360$ & 400 & 57.624 & 87.299 & 48.395 & 86.811 & 86.81 & N/A \\
& $300 \times 400$ & 300 & 103.62 & 145.09 & 86.889 & 144.59 & 144.42 & N/A \\
\hline \multirow{6}{*}{$X_{<>1}$} & $60 \times 80$ & $10^{4}$ & 0.35994 & 0.17525 & 0.30215 & 0.23642 & 0.241 & N/A \\
& $90 \times 120$ & $10^{4}$ & 1.5546 & 0.75564 & 1.3259 & 0.96344 & 0.9779 & N/A \\
& $120 \times 160$ & 6000 & 4.7353 & 2.3036 & 4.1074 & 2.8295 & 2.8673 & N/A \\
& $150 \times 200$ & 3000 & 10.88 & 5.2769 & 9.5609 & 6.3573 & 6.4427 & N/A \\
& $180 \times 240$ & 1500 & 23.226 & 11.274 & 20.643 & 13.339 & 13.467 & N/A \\
& $210 \times 280$ & 800 & 47.132 & 23.022 & 42.209 & 26.941 & 27.211 & N/A \\
& $240 \times 320$ & 500 & 92.218 & 44.629 & 82.921 & 51.535 & 51.874 & N/A \\
& $270 \times 360$ & 400 & 135.3 & 66.833 & 122.56 & 76.469 & 77.04 & N/A \\
& $300 \times 400$ & 300 & 231.45 & 111.57 & 210.66 & 127.03 & 128.03 & N/A \\
\hline
\end{tabular}

It is also worth comparing the obtained results with the ones published in [6]:

- the algorithm in [6] does not manage feasible regions of the kind $X_{<}$or $X_{<>}$;

- in [6] the change of vertex iterative steps are made numerically by means of the use of the "linsolve()" MATLAB command, this allows to solve a wide class of problems but is inherently slower than the simplex table approach proposed in this paper.

\section{Conclusion}

A class of generalized fractional programming problems is studied: the proposed objective function is the ratio of powers of affine functions and the feasible region is a polyhedron, not necessarily bounded. Despite its generality, the problem can be efficiently solved by means of a simplex-like method. The proposed algorithm has been implemented and validated by solving various instances, with a grand total of 520000 problems. The numerical tests have pointed out that the suggested algorithm benefits from the generalized convexity properties of the problem. It can also be underlined that, in managing the change of vertex iterative steps, the use of simplex table and pivot operations results to be very fast, allowing to solve large problems in a reasonable time. Moreover, unlike the standard linear fractional case, in the pseudoconcave case, the optimal value may not be attained at a vertex. 
Table 3 Computational Results - Real Times (secs)

\begin{tabular}{|c|c|c|c|c|c|c|c|c|}
\hline$X$ & $m \times n$ & num & $\begin{array}{l}\alpha=2 \\
\beta=3\end{array}$ & $\begin{array}{l}\alpha=3 \\
\beta=5\end{array}$ & $\begin{array}{l}\alpha=1 / 3 \\
\beta=3 / 4\end{array}$ & $\begin{array}{l}\alpha=3 \\
\beta=2\end{array}$ & $\begin{array}{l}\alpha=5 \\
\beta=3\end{array}$ & $\begin{array}{l}\alpha=1 / 2 \\
\beta=1 / 4\end{array}$ \\
\hline \multirow{9}{*}{$X_{>}$} & $60 \times 80$ & $10^{4}$ & 0.038445 & 0.03621 & 0.030776 & 0.061092 & 0.063556 & 0.067042 \\
\hline & $90 \times 120$ & $10^{4}$ & 0.16137 & 0.15191 & 0.12738 & 0.23795 & 0.24551 & 0.25831 \\
\hline & $120 \times 160$ & 6000 & 0.49226 & 0.46577 & 0.39143 & 0.69058 & 0.71108 & 0.7427 \\
\hline & $150 \times 200$ & 3000 & 1.1362 & 1.0781 & 0.91434 & 1.5382 & 1.576 & 1.635 \\
\hline & $180 \times 240$ & 1500 & 2.4205 & 2.304 & 1.9665 & 3.2015 & 3.2875 & 3.4054 \\
\hline & $210 \times 280$ & 800 & 5.0267 & 4.7959 & 4.1094 & 6.5247 & 6.6835 & 6.9148 \\
\hline & $240 \times 320$ & 500 & 9.9537 & 9.5244 & 8.2744 & 12.638 & 12.973 & 13.294 \\
\hline & $270 \times 360$ & 400 & 14.787 & 14.11 & 12.311 & 18.604 & 18.979 & 19.554 \\
\hline & $300 \times 400$ & 300 & 24.883 & 23.777 & 20.837 & 31.125 & 31.6 & 32.718 \\
\hline \multirow{9}{*}{$X_{<}$} & $60 \times 80$ & $10^{4}$ & 0.038359 & 0.080028 & 0.030789 & 0.078864 & 0.078844 & $\mathrm{~N} / \mathrm{A}$ \\
\hline & $90 \times 120$ & $10^{4}$ & 0.16003 & 0.31279 & 0.12652 & 0.30989 & 0.30969 & $\mathrm{~N} / \mathrm{A}$ \\
\hline & $120 \times 160$ & 6000 & 0.49122 & 0.88434 & 0.39087 & 0.87883 & 0.87866 & $\mathrm{~N} / \mathrm{A}$ \\
\hline & $150 \times 200$ & 3000 & 1.1492 & 1.9353 & 0.92373 & 1.9241 & 1.9238 & $\mathrm{~N} / \mathrm{A}$ \\
\hline & $180 \times 240$ & 1500 & 2.4708 & 3.9529 & 2.0052 & 3.9375 & 3.9365 & $\mathrm{~N} / \mathrm{A}$ \\
\hline & $210 \times 280$ & 800 & 5.0731 & 7.9267 & 4.1618 & 7.8935 & 7.8989 & $\mathrm{~N} / \mathrm{A}$ \\
\hline & $240 \times 320$ & 500 & 10.128 & 15.185 & 8.3509 & 15.112 & 15.116 & $\mathrm{~N} / \mathrm{A}$ \\
\hline & $270 \times 360$ & 400 & 14.632 & 22.117 & 12.302 & 21.993 & 21.994 & $\mathrm{~N} / \mathrm{A}$ \\
\hline & $300 \times 400$ & 300 & 26.289 & 36.75 & 22.063 & 36.627 & 36.582 & $\mathrm{~N} / \mathrm{A}$ \\
\hline \multirow{9}{*}{$X_{<>}$} & $60 \times 80$ & $10^{4}$ & 0.098159 & 0.049703 & 0.083661 & 0.066276 & 0.067521 & $\mathrm{~N} / \mathrm{A}$ \\
\hline & $90 \times 120$ & $10^{4}$ & 0.40829 & 0.20187 & 0.35058 & 0.25601 & 0.25976 & $\mathrm{~N} / \mathrm{A}$ \\
\hline & $120 \times 160$ & 6000 & 1.2229 & 0.6009 & 1.0649 & 0.7357 & 0.74532 & $\mathrm{~N} / \mathrm{A}$ \\
\hline & $150 \times 200$ & 3000 & 2.7863 & 1.3615 & 2.4551 & 1.6361 & 1.6576 & $\mathrm{~N} / \mathrm{A}$ \\
\hline & $180 \times 240$ & 1500 & 5.8831 & 2.8683 & 5.2364 & 3.3901 & 3.422 & $\mathrm{~N} / \mathrm{A}$ \\
\hline & $210 \times 280$ & 800 & 12.003 & 5.8801 & 10.759 & 6.8767 & 6.9459 & $\mathrm{~N} / \mathrm{A}$ \\
\hline & $240 \times 320$ & 500 & 23.608 & 11.45 & 21.24 & 13.215 & 13.301 & $\mathrm{~N} / \mathrm{A}$ \\
\hline & $270 \times 360$ & 400 & 34.289 & 16.97 & 31.073 & 19.411 & 19.556 & $\mathrm{~N} / \mathrm{A}$ \\
\hline & $300 \times 400$ & 300 & 58.646 & 28.308 & 53.396 & 32.228 & 32.48 & $\mathrm{~N} / \mathrm{A}$ \\
\hline
\end{tabular}

Acknowledgements We would like to thank the anonymous referees and Prof. Alberto Cambini for their helpful comments and discussions.

\section{Appendix}

In this appendix a detailed description of the algorithm is presented. In particular, the main procedure is aimed to initialize the process and to split the visit of the feasible region with respect to the sign of the linear function $c^{T} x+c_{0}$. As we need to follow different steps according to the generalized convexity property of $f$, we will distinguish two subprocedures: Visit1 finds maximum points when $f$ is pseudoconcave, while Visit2 deals with the pseudoconvex case. It's worth remarking that we consider the case $\alpha \neq \beta$, since for $\alpha=\beta$, solution methods have been already proposed (see for instance [5]).

Denote by $x_{b}$ an incumbent maximum point of $\mathrm{P}$ and by $U_{b}$ the incumbent optimal value of $\mathrm{P}$. Set:

$$
\begin{array}{ll}
C_{\max }=\sup _{x \in X}\left(c^{T} x+c_{0}\right) & C_{\min }=\inf _{x \in X}\left(c^{T} x+c_{0}\right) \\
\theta_{\min }=\min _{x \in X}\left(d^{T} x+d_{0}\right) & \theta_{\max }=\sup _{x \in X} d^{T} x+d_{0} \\
X_{<}=\left\{x \in X: c^{T} x+c_{0}<0\right\} &
\end{array}
$$

We are ready to present the main algorithm and the two subprocedures Visit1 and Visit2; to get them more readable, some comment rows have been added. 


\section{Main algorithm.}

Compute $C_{\max }$ and $C_{\min }$

$U_{b}=-\infty$ and $x_{b}=\emptyset$

If $C_{\min } C_{\max } \leq 0$, then

$\sharp$ The optimal value of $f$ is at least $0 \sharp$

$U_{b}=0$ and $x_{b}$ is a zero of $c^{T} x+c_{0}$ on $X$

end if

If $C_{\max }>0$, then

$\sharp$ we find the optimal solution of $f$ on $C^{+} \cap X \sharp$

if $\alpha>\beta$, then

$\sharp$ In this case $f$ is pseudoconvex $\sharp$

if $C_{\max }=+\infty$, then $\sup P=+\infty$, STOP

else $\left[x_{b}, U_{b}\right]=\operatorname{Visit} 2\left(x_{b}, U_{b}, c, c_{0}, C^{+}\right)$

end if

else

$\sharp$ In this case $f$ is pseudoconcave $\sharp$

$\left[x_{b}, U_{b}\right]=\operatorname{Visit1}\left(x_{b}, U_{b}, c, c_{0}, C^{+}\right)$

end if

else

if $\left(C_{\max }\right)^{\alpha}>0$, then

$\sharp$ In this case $X=X_{<}$; we find the maximum of $f$ on $C^{-} \cap X$.

In $P(\theta)$ we substitute $c^{T} x+c_{0}$ with $-\left(c^{T} x+c_{0}\right) \sharp$

if $\alpha>\beta$, then $\left[x_{b}, U_{b}\right]=\operatorname{Visit} 2\left(x_{b}, U_{b},-c,-c_{0}, X_{<}\right) \sharp P$ seudoconvex case $\sharp$

else $\left[x_{b}, U_{b}\right]=\operatorname{Visit} 1\left(x_{b}, U_{b},-c,-c_{0}, X_{<}\right) \sharp$ Pseudoconcave case $\sharp$

end if

end if

end if

If $C_{\min }<0$

$\sharp$ we find the optimal solution of $f$ on $X<\sharp$

if $\left(C_{\min }\right)^{\alpha}>0$ and $C_{\max }>0$, then

$\sharp$ In this case $f$ is positive and the numerator changes sign. We have already found the maximum value on $C^{+} \cap X$. We find the optimal value of $f$ on $X<\cap X$ and we find the global optimum by comparing the two values. $\sharp$

if $\alpha>\beta$, then $\left[x_{b}, U_{b}\right]=\operatorname{Visit} 2\left(x_{b}, U_{b},-c,-c_{0}, X_{<}\right)$

else $\left[x_{b}, U_{b}\right]=\operatorname{Visit} 1\left(x_{b}, U_{b},-c,-c_{0}, X_{<}\right)$

end if

else if $U_{b}<0$, then

$\sharp f$ is always negative, in this case $X \subset S^{-} \sharp$

if $\alpha<\beta$ then $\left[x_{b}, U_{b}\right]=\operatorname{Visit} 2\left(x_{b}, U_{b}, c, c_{0}, X_{<}\right) \sharp$ Pseudoconvex case $\sharp$

else $\left[x_{b}, U_{b}\right]=\operatorname{Visit} 1\left(x_{b}, U_{b}, c, c_{0}, X_{<}\right) \sharp$ Pseudoconcave case $\sharp$

end if

end if

end if

\section{end Main algorithm}


function $\left[x_{b}, U_{b}\right]=\operatorname{Visit1}\left(x b, U B, C, C_{0}, W\right) \sharp$ Pseudoconcave case. $\sharp$ $\sharp$ with respect to the calls in the main algorithm, $C$ will be either $c$ or $-c, C_{0}$ will be either $c_{0}$ or $-c_{0}$, while $W$ will be either $C^{+}$or $X_{<\cdot \sharp}$

ended $=$ false

\# "ended" will take value true as soon as the iterative cycle "while" has to be stopped \# Determine $\theta_{\min }$ and solve

$\hat{P}_{c}: \max \left(C^{T} x+C_{0}\right), x \in X \cap W \cap\left\{x: d^{T} x+d_{0}=\theta_{\min }\right\}$

If $\alpha \leq \beta$ and $\hat{P}_{c}$ has no optimal solutions, then $\sup P=+\infty$, STOP else

determine $\theta_{\max }$, let $\theta_{\max }=\theta_{\max }-\theta_{\min }$

let $x_{0}$ be an optimal solution of $\hat{P}_{c} \sharp$ it is also an optimal solution of $\hat{P}\left(\theta_{0}\right): \max \left(C^{T} x+C_{0}\right), x \in X \cap W \cap\left\{x: d^{T} x+d_{0}=\theta_{0}\right\}$ with $\theta_{0}=\theta_{\text {min }} \sharp$ set $k=0$

while not(ended) do

Determine the stability interval $\left[\theta_{k}, \theta_{k+1}\right]$ associated with the optimal solution $x\left(\theta_{k}\right)=\left(x_{B_{k}}+\theta_{k} u_{B_{k}}, 0\right)$ of $P\left(\theta_{k}\right)$

Compute $h^{\prime}\left(\theta_{k}\right)$

If $h^{\prime}\left(\theta_{k}\right) \leq 0$, then

if $U_{b}<h\left(\theta_{k}\right)$, then $U_{b}=h\left(\theta_{k}\right), x_{b}=x\left(\theta_{k}\right)$ end if

$\sharp$ the optimal value is updated and the incumbent optimal point $x_{b}$ corresponds to a vertex of the feasible region $\sharp$

ended $=$ true

else $\widetilde{\theta}=\frac{\xi_{B_{k}}}{(\beta-\alpha) c_{B_{k}}^{T} u_{B_{k}}}$

if $\theta_{k}<\widetilde{\theta} \leq \theta_{k+1}$ then

if $U_{b}<h\left(\theta_{k}\right)$, then $U_{b}=h\left(\theta_{k}\right), x_{b}=x\left(\theta_{k}\right)$ end if

$\sharp$ the optimal value is updated, $x_{b}$ does not correspond to a vertex $\sharp$ ended $=$ true

else

if $\alpha>\beta$ and $\theta_{k+1}=+\infty$, then ended $=$ true, STOP: $\sup P=0$

$\sharp$ In this case $f$ is always negative and there exists an extreme direction $d$ and a point $x_{0}$ such that $\lim _{t \rightarrow+\infty} \frac{\left(c^{T} x_{0}+t c^{T} u+c_{0}\right)^{\alpha}}{\left(d^{T} x_{0}+t d^{T} u+d_{0}\right)^{\beta}}=0 \sharp$

else

If $\theta_{k+1} \geq \theta_{\max }$, then

if $U_{b}<h\left(\theta_{k+1}\right)$, then $U_{b}=h\left(\theta_{k+1}\right), x_{b}=x\left(\theta_{k+1}\right)$ end if $\sharp$ the optimal value is updated and the incumbent optimal point $x_{b}$ corresponds to $\theta_{\max }$, that is to a vertex of the feasible region $\sharp$ ended $=$ true

else

let $i$ such that $x_{B_{k_{i}}}+\theta_{k+1} u_{B_{k_{i}}}=0$

Perform a pivot operation by means of the dual simplex algorithm, set $k=k+1$

end if

end if

end if 
end if

end do

end if

\section{end function Visit1}

function $\left[x_{b}, U_{b}\right]=\operatorname{Visit2}\left(x_{b}, U_{b}, C, C_{0}, W\right) \sharp$ Pseudoconvex case. $\sharp$

$\sharp$ with respect to the calls in the main algorithm, $C$ will be either $c$ or $-c, C_{0}$ will be either $c_{0}$ or $-c_{0}$, while $W$ will be either $C^{+}$or $X_{<\cdot \sharp}$

Determine $\theta_{\max }$

If $\alpha<\beta$ and $\theta_{\max }=+\infty$, then $\sup P=0, \mathrm{STOP}$

else

ended $=$ false

\# "ended" will take value true as soon as the iterative cycle "while" has to be stopped $\sharp$

if $\theta_{\max }=+\infty$, then $\theta_{\max }=\operatorname{Big} M$ end if

determine $\theta_{\min }$ and set $\theta_{\max }=\theta_{\max }-\theta_{\min }$

solve $\hat{P}_{c}: \max \left(C^{T} x+C_{0}\right), x \in X \cap W \cap\left\{x: d^{T} x+d_{0}=\theta_{\min }\right\}$

let $x_{0}$ be an optimal solution of $\hat{P}_{c} \sharp$ it is also an optimal solution of

$\hat{P}\left(\theta_{0}\right): \max \left(C^{T} x+C_{0}\right), x \in X \cap W \cap\left\{x: d^{T} x+d_{0}=\theta_{0}\right\}$ with $\theta_{0}=\theta_{\min }$.

set $k=0$

while not(ended) do

determine the stability interval $\left[\theta_{k}, \theta_{k+1}\right]$ associated with the optimal solution $x\left(\theta_{k}\right)=\left(x_{B_{k}}\left(\theta_{k}\right), 0\right)=\left(x_{B_{k}}+\theta_{k} u_{B_{k}}, 0\right)$ of $P\left(\theta_{k}\right)$

compute $h^{\prime}\left(\theta_{k}\right)$

if $h^{\prime}\left(\theta_{k}\right)<0$, then

$\sharp x\left(\theta_{k}\right)$ is a local maximum point for $f \sharp$

if $U_{b}<h\left(\theta_{k}\right)$, then $U_{b}=h\left(\theta_{k}\right), x_{b}=x\left(\theta_{k}\right)$ end if

if $h^{\prime}(\theta)<0, \forall \theta>\theta_{k}$, then ended=true, STOP

$\sharp$ the optimal value is updated and the incumbent optimal point $x_{b}$ corresponds to $\theta_{k}$, that is to a vertex of the feasible region $\sharp$

else

$\sharp x\left(\theta_{k}\right)$ is a local maximum point, maybe different from the global one. $\sharp$ $\sharp j u m p \sharp$

find $\widetilde{\theta}_{k}$ such that $h\left(\theta_{k}\right)=h\left(\widetilde{\theta}_{k}\right)$

if $\widetilde{\theta}_{k} \leq \theta_{k+1}$, then

$\sharp$ the value of $f$ increases up to $\theta_{k+1}, x\left(\theta_{k}\right)$ is a local maximum point $\sharp$ if $U_{b}<h\left(\theta_{k+1}\right)$, then $U_{b}=h\left(\theta_{k+1}\right), x_{b}=x\left(\theta_{k+1}\right)$ end if $\sharp$ the optimal value is updated and the incumbent optimal point $x_{b}$ corresponds to $\theta_{k+1} \sharp$

if $\theta_{k+1}=\theta_{\max }$, then ended $=$ true

else

let $i$ be such that $x_{B_{i}}+\theta_{k+1} u_{B_{k_{i}}}=0$

perform a pivot operation by means of the dual simplex algorithm and find a new basis solution $x\left(\theta_{k+1}\right)$ set $k=k+1$ 
end if

else

if $\widetilde{\theta}_{k}>\theta_{\max }$, then

if $U_{b}<h\left(\theta_{k}\right)$, then $U_{b}=h\left(\theta_{k}\right), x_{b}=x\left(\theta_{k}\right)$ end if

$\sharp$ the optimal value is updated and the incumbent optimal point

$x_{b}$ corresponds to $\theta_{k} \sharp$

ended $=$ true

else

$\sharp$ feasibility is lost. It has to be restored by solving a new parametric problem $\hat{P}_{c} \sharp$

$\theta_{\max }=\theta_{\max }-\widetilde{\theta}_{k}, \theta=\theta+\widetilde{\theta}_{k}, h(\theta)=h\left(\theta+\widetilde{\theta}_{k}\right)$

solve

$\hat{P}_{c}: \max \left(C^{T} x+C_{0}\right)$

$$
x \in X \cap W \cap\left\{x: d^{T} x+d_{0}=\theta_{\min }+\widetilde{\theta}_{k}\right\}
$$

end if

end if

end if

else

if $\theta_{k+1}=\theta_{\max }$, then

ended $=$ true

$\sharp$ the optimal value is updated and the incumbent optimal point $x_{b}$ corresponds to $\theta_{k} \sharp$

if $U_{b}<h\left(\theta_{k+1}\right)$, then $U_{b}=h\left(\theta_{k+1}\right), x_{b}=x\left(\theta_{k+1}\right)$ end if

else

let $i$ be such that $x_{B_{i}}+\theta_{k+1} u_{B_{k_{i}}}=0$

performing a pivot operation by means of the dual simplex algoritm and find a new basis solution $x\left(\theta_{k+1}\right)$

set $k=k+1$

end if

end if

end do

end if

\section{References}

1. Cambini, A., Martein, L., A modified version of Martos' algorithm, Methods of Operation Research, 53, 33-44, (1986).

2. Cambini, A., Martein, L., Linear Fractional and Bicriteria Linear Fractional Programs. In Cambini, A., Castagnoli, E., Martein, L., Mazzoleni, P., Schaible, S. (eds) Generalized Convexity and Fractional Programming with Economic Applications, 345, 155-166, Springer, Heidelberg (1990), ISBN: 0-387-52673-0.

3. Cambini, A., Martein, L., Generalized Convexity and Optimization: Theory and Applications, 616, Springer, Heidelberg (2009), ISBN: 978-3-540-70875-9.

4. Cambini, R., A class of non-linear programs: theoretical and algorithmical results. In Komlósi, S., Rapcsák T., Schaible S. (eds.)Generalized Convexity, 405, Springer-Verlag, Berlin Heidelberg, 294-310, (1994), ISBN: 3-540-57624-X. 
5. Cambini R., Sodini, C., A unifying approach to solve some classes of rank-three multiplicative and fractional programs involving linear functions. European Journal of Operation Research 207, 25-29 (2010). ISSN 0377-2217.

6. Cambini R., Sodini, C., Global optimization of a rank-two nonconvex program. Mathematical Methods of Operations Research 71, 1, 165-180 (2010). ISSN: 1432-2994.

7. Carosi, L., Martein, L., A sequential method for a class of pseudoconcave fractional problems. Central European Journal of Operations Research 16, 2, 153-164 (2008).

8. Dai, Z., Wu, Y., Zhang, F., Wang, H., A novel fast method for $L^{\infty}$ problems in multiview geometry. In Fitzgibbon, A., Lazebnik, S., Perona, P., Sato, Y., Schmid, C., (Eds.), Computer Vision-ECCV 2012, 12 th European Conference on Computer Vision, Florence, Italy, October 7-13, 2012, Proceedings, Part V, 116-129, Springer Berlin Heidelberg (2012)

9. Ellero, A., The optimal level solutions method, Journal of Information and Optimization Sciences 17, 2, 355-372 (1996).

10. Frenk, J.B.G., Schaible, S., Fractional programming. In Hadjisavvas, N., Komlósi, S., Schaible S. (eds.) Handbook of Generalized Convexity and Generalized Monotonicity, pp. 335 - 386, Springer, New York (2005)

11. Stancu-Minasian, I.M., Fractional Programming Theory, Methods, and Applications. Kluwer Academic Publisher, New York (1997), ISBN: 978-0-7923-4580-0.

12. Stancu-Minasian, I. M., A sixth bibliography of fractional programming. Optimization, 55, 4, 405-428 (2006). 